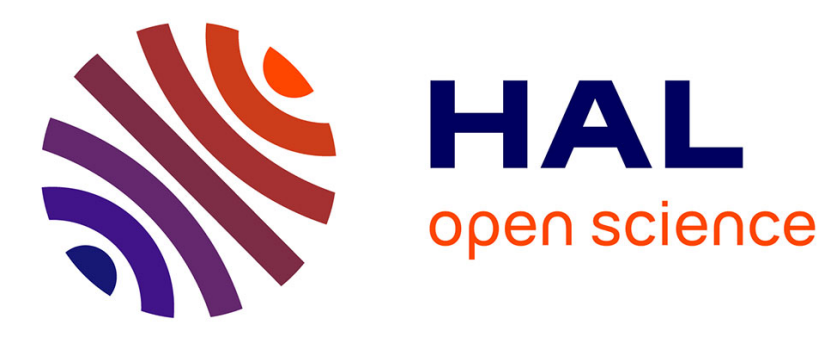

\title{
Choice Modeling for Usage Context-Based Design
}

Lin He, Wei Chen, Christopher Hoyle, Bernard Yannou

\section{To cite this version:}

Lin He, Wei Chen, Christopher Hoyle, Bernard Yannou. Choice Modeling for Usage Context-Based Design. Journal of Mechanical Design, 2012, 134 (3), pp.1-26. 10.1115/1.4005860 . hal-01152304

\section{HAL Id: hal-01152304 \\ https://hal-centralesupelec.archives-ouvertes.fr/hal-01152304}

Submitted on 15 May 2015

HAL is a multi-disciplinary open access archive for the deposit and dissemination of scientific research documents, whether they are published or not. The documents may come from teaching and research institutions in France or abroad, or from public or private research centers.
L'archive ouverte pluridisciplinaire HAL, est destinée au dépôt et à la diffusion de documents scientifiques de niveau recherche, publiés ou non, émanant des établissements d'enseignement et de recherche français ou étrangers, des laboratoires publics ou privés. 


\title{
Choice Modeling for Usage Context-Based Design
}

\author{
Lin $\mathrm{He}$ \\ Graduate Student \\ Mechanical Engineering \\ Northwestern University \\ 2145 Sheridan Road, Tech B224 \\ Evanston, IL 60208
}

\author{
Wei Chen* \\ Professor \\ Mechanical Engineering \\ Northwestern University \\ 2145 Sheridan Road, Tech B224 \\ Evanston, IL 60208 \\ weichen@northwestern.edu

Bernard Yannou
Professor
Ecole Centrale Paris
Laboratoire Genie Industriel
Grande Voie des Vignes
92290, Chatenay-Malabry, France

\begin{abstract}
Usage Context-Based Design (UCBD) is an area of growing interest within the design community. Usage context is the set of scenarios in which a product (or service) is to be used, including the environments in which the product is used, the types of tasks the product performs, and the conditions under which the product is purchased and operates. It is proposed in this work that in choice modeling for usage context-based design, usage context should be a part of the primary descriptors in the definition of a customer profile, in addition to the socio-demographic attributes for modeling customers' heterogeneity. As customers become more technology-savvy and market-educated, current choice modeling methods in engineering design could greatly benefit from exploiting the rich contextual information existing in product usage. In this work, we propose a choice modeling framework for Usage Context-based Design (UCBD) to quantify the impact of usage context on customer choices. We start with defining a taxonomy for UCBD. By explicitly modeling usage context's influence on both product performances and customer preferences, a step-by-step choice modeling procedure is proposed to support UCBD. Two case studies, a jigsaw example with stated preference data and a hybrid electric vehicle example with revealed preference data, demonstrate the needs and benefits of incorporating usage context in choice modeling.
\end{abstract}




$\begin{array}{ll}\text { NOMENCLATURE } \\ \mathbf{A} & \text { Customer-desired product attributes } \\ \beta & \text { Coefficients in customer's choice utility function } \\ \mathbf{C V} & \text { Conventional vehicle } \\ \text { DCA } & \text { Discrete Choice Analysis } \\ \text { DOE } & \text { Design of experiment } \\ \mathbf{E} & \text { Usage context attributes } \\ \mathbf{E}_{\mathbf{W}} & \text { Preference-related usage context attributes } \\ \mathbf{E}_{\mathbf{Y}} & \text { Performance-related usage context attributes } \\ \boldsymbol{\varepsilon}_{n i} & \text { Random disturbance of customer choice utility of product } i \text { by customer } n \\ \text { HEV } & \text { Hybrid electric vehicle } \\ \mathbf{k} & \text { Cut points in ordered logit model } \\ \mathbf{M} & \text { Exogenous variables (representing sources of uncertainty in market) } \\ \mathbf{M N L} & \text { Multinomial logit } \\ P_{n i} & \text { Choice probability for product } i \text { and customer } n \\ \text { PHEV } & \text { Plug-in hybrid electric vehicle } \\ \mathbf{R} & \text { Subjective ratings } \\ \mathbf{S} & \text { Customer profile attributes } \\ \mathbf{S}_{\mathbf{W}} & \text { Preference-related customer profile attributes } \\ \mathbf{S} & \text { Performance-related customer profile attributes } \\ u_{n i} & \text { Customer choice utility of product } i \text { by customer } n \\ \text { UCBCM } & \text { Usage context-based choice modeling } \\ \text { UCBD } & \text { Usage context-based design } \\ W_{n i} & \text { Observed (deterministic) part of the customer choice utility of product } i \text { by } \\ \mathbf{X} & \text { customer } n \\ \mathbf{Y} & \text { Engineering design options or variables } \\ \mathbf{Y}_{\mathbf{r}} & \text { Engineering performance } \\ \mathbf{Y}_{\mathbf{t}} & \text { Performance of service results } \\ & \text { Performance of transformation } \\ & \end{array}$

\section{INTRODUCTION}

Customer choice (demand) modeling is becoming prevalent in engineering design research for designing products or product families [1-13]. Accurately capturing customer choice is essential because it allows for the prediction of future product demand as a function of engineering design across a heterogeneous customer population, characterized by multiple market segments. Capturing heterogeneous choice behavior is achieved using disaggregate demand modeling methods, with the probabilistic Discrete Choice Analysis (DCA) [14] method being the most widely used approach used in engineering design. Depending upon the degree of heterogeneity and the specific design problem, different types of DCA models, such as multinomial logit models [15], nested logit models [16], and mixed logit models [17], have been utilized in design to capture heterogeneity in customer preferences. For instance, by allowing random taste variation across the population using a Hierarchical Bayes mixed logit model, Michalek [4] modeled heterogeneity in customer preference using random model parameters (i.e. random heterogeneity) without including the customer profile into choice modeling. Sullivan et al. [7] 
compared continuous representations of customer heterogeneity using Hierarchical Bayes mixed logit models with discrete representations using Latent Class mixed logit models where customers are grouped into segments based on their preferences; several limitations of the Latent Class mixed logit models for fully capturing preference behavior were identified. By introducing the customer profile attributes as explicit terms in the choice utility function, Hoyle et al. [18] introduced systematic customer heterogeneity, in addition to random heterogeneity, into a hierarchical choice modeling framework using a Hierarchical Bayes mixed logit model.

While previous works have laid the foundation for modeling the heterogeneity in consumers' choice behavior, the potential of disaggregate choice modeling in engineering design has not been fully realized due to an overreliance on marketing and demographic attributes (gender, age, income etc.) to approximate the complex drivers behind heterogeneous customer choice. Existing choice modeling methods lose their effectiveness and fail to offer insights into why choices were made, because of the limited scope of customer attributes included in the model. For this reason, it is necessary to investigate the reasons behind and the situations under which a product is being used to fully understand and model heterogeneous choice behavior. Hence, we must delve into a more proactive modeling approach to discover driving factors underlying customers' choices by taking into account the usage context of a product. In this work, the usage context of a product is defined as "all aspects describing the context of product use that vary under different use conditions and affect product performance and/or consumer preferences for the product attributes". The usage context may also have a significant impact on the product performance, which is not considered in existing methods that simply treat product performance as "constant" across all consumers and usage contexts in choice modeling.

Marketing researchers were among the first to recognize the importance of product usage context. As Belk pointed out, use situation has "a demonstrable and systematic effect on current behavior" [19]. Dickson [20] proposed a person-situation (usage context in our terminology) framework in which the market is explicitly segmented by groups of consumers within usage situations. More recently, De la fuente and Guillen [21] studied the usage suitability of household cleaning products and their influences on purchase behavior. Although existing literature illustrated the significance of usage context in the consumers' choice process, the linkage between usage context and product performance as well as product design is missing, which calls for an innovative way to explicitly model usage context's impact with analytical methods.

In this work, we propose the founding principles underlying a choice modeling approach to Usage Context-based Design (UCBD), where usage context is considered as a critical part of driving factors behind customers' choice, in addition to customer demographic attributes and product design attributes. In this paper, we first provide a review of usage context influence, based on the literature from both market research and engineering design (Section 2). A taxonomy for UCBD is proposed in Section 3 by defining the important terms and their relations. Next, we discuss how the taxonomy is integrated into a step-by-step choice modeling procedure to support UCBD which captures the impact of usage context by explicitly modeling its influence on both product performances and customer preferences (Section 4). Findings from both a jigsaw case study with stated preference data (Section 5) and a hybrid electric vehicle (HEV) case study with revealed preference data (Section 6) demonstrate the needs and benefits of incorporating usage context in choice modeling. Conclusions and future work are summarized at the end. 


\section{LITERATURE ON USAGE CONTEXT STUDY}

\subsection{Usage context literature in market research}

Marketing researchers have long been interested in understanding and conceptualizing the underlying factors behind customer behavior and therefore are among the first to recognize the power of situational (usage contextual) influence on behavior [22-24]. Belk [19] laid out the definition of use situation as follows: "all those factors particular to a time and place of observation which do not follow from a knowledge of personal (intra-individual) and stimulus (choice alternative) attributes, and which have a demonstrable and systematic effect on current behavior." Belk later proposed a revised stimulus-organism-response (S-O-R) paradigm [25] in which the stimulus is divided into an object and a situation, or usage context in our terminology. Relating to Belk's S-O-R paradigm, we propose here an UCBD influence diagram as illustrated in Figure 1.

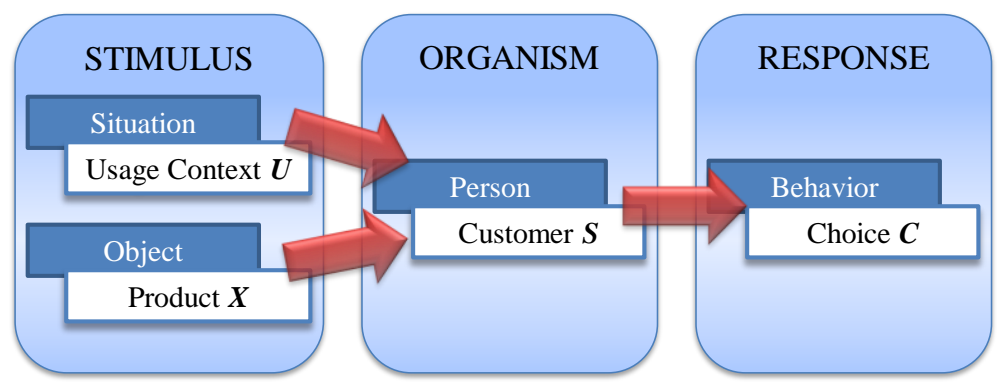

Figure 1: UCBD Influence Diagram based on Belk's S-O-R Paradigm

In the context of UCBD, object refers to product and situation refers to usage context. Both usage context and product act as stimulus to a customer which leads to his/her choice behavior. Besides the conceptualization, Belk's categorization of five groups of situational characteristics (named usage context attributes $\mathbf{E}$ in this work) [25] serves as the foundation for developing and classifying the usage context attributes for choice modeling; more details on this subject will be provided in Section 3.

The need for considering situational (usage contextual) variables in market segmentation was first recognized in the 1980s. Dickson [20] pointed out that usage situation is overlooked in market segmentation and presented a person-situation segmentation framework in which the market is explicitly segmented by groups of consumers within usage situations. The work by Christensen et al. [26] recommends ending the common practice of segmenting customers based on their demographics and replacing it with ways that reflect how customers actually live their lives. The "substitution in use" (SIU) approach by Stefflre [27] was developed based on the premise that consumers think about product category instances within their functional roles in various possible usage contexts. As a further validation of this premise, in a more recent case study of snack foods, Ratneshwar and Shocker [28] showed that products which do not belong to the same category could be considered as comparable in certain usage contexts, highlighting the need for constructing different choice set alternatives based on customer profile and usage context. More recently, De la fuente and Guillen [21] analyzed consumer perceptions with regard to the suitability of household cleaning products to anticipated usage contexts, as well as their influences on purchase behavior. In the case of multiple usage context scenarios, Berkowitz, Ginter and Talarzyk [29] suggested aggregating an individual's given usage situation demand weighted by the situation's frequency of occurrence or importance. While their approach 
demonstrated the influence of usage suitability on consumer choice, the linkage between usage context and product performance, as well as product design is absent.

\subsection{Usage context literature in engineering design}

Even though the study of usage context in consumer behavior has been prevalent for years, it had not been applied to engineering design until 1990s. In Ulrich and Eppinger's product design and development book [30], the need for designers to envision a product's "use environment" in identifying customer needs is emphasized. Methods have been suggested to observe a product in use as a way of gathering raw data from customers. More recently, Green et al. published three successive papers [31-33] on a frontier design method for product usage context, which is defined as a combination of application and environment in which a product will be used. A broader concept of product design context is constructed, consisting of three contexts that influence customer preferences: usage context, customer context and market context. Their work supports the idea that context can be differentiated based upon functional attributes, indicating a link between engineering parameters and perceived usefulness, which occurs under the influence of different usage contexts. Most recently, study on usage context attributes of Hybrid Electric Vehicles [34] suggested that usage context should be treated as an additional dimension of the customer characterization process to reflect their preference heterogeneity.

While previous work introduced usage context-based design, their findings on usage context are mainly focused on qualitative analysis to support concept generation. However, the benefits of understanding usage context have great potential in an analytical design process as well. Through a choice model, we can understand the impact that usage context has on product performance and customer preferences, and therefore optimize product design to maximize the customer demand, or profit contributed by the product. In this work, we propose a comprehensive choice modeling framework for Usage Context-Based Design (UCBD) to quantitatively incorporate usage context into the product design process. In the following sections, a taxonomy for UCBD is first provided and is followed by a presentation of the fourphase choice modeling procedure, illustrated with two case studies.

\section{TAXONOMOY IN USAGE CONTEXT-BASED DESIGN}

As shown in Section 2, previous works in marketing research and product design have employed different definitions and terminologies for usage context related variables. For instance, usage context is also called use situation; a usage context attribute is also referred to as a situational variable. To establish a common foundation for choice modeling in UCBD, we devote this section to defining a taxonomy by following the established classification in the market research domain and the specific needs associated with product design. To illustrate the taxonomy, the design of a jigsaw for cutting wood is used as an example throughout this section.

\section{Usage context attributes $-\mathbf{E}$}

Usage context attributes $\mathbf{E}$ refer to the characteristics or attributes used to describe the usage context. Associated with this taxonomy is the definition of "usage context". Belk [19] stated that use situation includes all factors that influence the customer behavior at a given time and place, except for the customer profile and product attributes. Unlike Belk, Green et al. [33] narrows down the scope of "usage context" to two major aspects, the application context and the environment context, and limits the influence of usage context to customer preferences only. Usage context in real life varies significantly across product categories. In our view, its influence on customer behavior includes the impact on product performance, choice set, and customer 
preference. Hence, we define the usage context in our work as "all aspects describing the context of product use that vary under different use conditions and affect product performance and/or consumer preferences for the product attributes". This definition emphasizes two concepts key to our approach. First, usage context covers all aspects related to the use of a product, but excludes customer profile (demographic attributes) and product attributes, which will be defined later on in this section. Second, usage context influences customer behavior through product performance, the choice set, as well as customer preference.

One consideration to note is that, under many circumstances, it is difficult to draw a clear distinction between the customer profile and usage context as separate sources of influence on customers' choice. In some cases, customer profile attributes may also seem like a usage context attribute, or vice versa. For example a customers' purchase history can be regarded either as a customer attribute or as a usage context. As a guideline, we refer to customer profile attributes as those stable characteristics of a customer that do not vary over a set of usage contexts, while those temporal, transitory characteristics of a customer that do vary over usage contexts belong to the area of usage context. In other words, these usage context attributes change from application to application or from environment to environment over time. For example, the skill of the customer to successfully accomplish a cutting task using a power tool, brand loyalty, and positive or negative experiences with a particular brand [35], are considered as customer profile attributes, since they are more stable over time than the usage context attributes.

Specific to the choice modeling process, we can divide usage context attributes $\mathbf{E}$ into performance-related and preference-related, according to the way in which they impact customer behavior. These usage context attributes $\mathbf{E}$ can be either continuous or discrete. While performance-related attributes $\mathbf{E}_{\mathbf{Y}}$ influence product performance $\mathbf{Y}$, preference-related attributes $\mathbf{E}_{\mathbf{W}}$ have an impact on the choice set and customer preference. In some cases, performancerelated and preference-related usage context attributes are not mutually exclusive. For example in using a jigsaw, if the saw is to be used for cutting outdoors, the density of saw dust experienced by the user may be different than if the saw were used indoors (performance impact), whereas the user may prefer a bright saw color for outdoor use so that the saw will be easily identified if placed on the ground (preference impact). Whether a usage context attribute is related to performance or preference can be determined by prior knowledge of experienced users or by the observations of products being used. Prior knowledge of a usage context attribute's influence on preference can be used to reduce the complexity of estimating a choice model, and hypothesis testing of a usage context attribute in the choice model estimation process can be used to confirm this knowledge.

\section{Customer profile attributes $-\mathbf{S}$}

The customer profile $\mathbf{S}$ includes all stable or permanent aspects of customer profile attributes impacting customer choice behavior, for example, gender, age, income bracket, etc. In the choice modeling of UCBD, customer profile attributes $\mathbf{S}$ may have a direct impact on customers' preference and therefore may influence their choices. Similar to usage context attributes, customer profile attributes $\mathbf{S}$ can be categorized into performance-related $\mathbf{S}_{\mathbf{Y}}$ and preferencerelated $\mathbf{S}_{\mathbf{W}}$ to differentiate their impact. For example, household income belongs to $\mathbf{S}_{\mathbf{W}}$, as it is expected to have a large impact on customers' sensitivity on price: the more they earn, the less they care about the price. On the other hand, skill level of the customer operating jigsaw is considered as a performance-related $\mathbf{S}_{\mathbf{Y}}$, because jigsaw performances vary when operated by a beginner, intermediate, or experienced user. 


\section{Customer desired product attributes $-\mathbf{A}$}

Customer-desired product attributes $\mathbf{A}$ are defined as key product characteristics that influence consumers' choices in selecting a product. In a market survey, consumers are usually asked to rate these customer-desired product attributes. They include not only engineering performances $\mathbf{Y}$, but also non-engineering attributes $\mathbf{M}$.

Engineering performance $\mathbf{Y}$ refers to all performance-related engineering attributes. Since $\mathbf{Y}$ plays a critical role in the engineering design process, engineering performance $\mathbf{Y}$ is our focus in this work. In the jigsaw example and other similar cases, engineering performance $\mathbf{Y}$ is further divided into performance of service results and performance of service delivery or transformations. The performance of service results $\mathbf{Y}_{\mathbf{r}}$ represents the measures of the end performances of the resulting service, such as cutting precision, planarity, etc. On the other hand, the performance of service delivery or transformations $\mathbf{Y}_{\mathbf{t}}$ represents the measures of the performances related to the delivery of the service, such as linear speed, noise, vibration, safety conditions, etc. The performances of the service delivery are no longer visible in the results once it has been delivered.

Non-engineering attributes $\mathbf{M}$ include all non-engineering aspects of customer desires attributes, for example, price, brand, aesthetics and other common marketing measures. Price is one of the most influential non-engineering attributes $\mathbf{M}$ in customers' choice. In practice, price can enter the utility function as a single term, or can be scaled by income or log income to reflect the connection between income and price sensitivity, as shown in the case study.

\section{Product choice set $-J_{n}$}

The product choice set $J_{n}$ is defined as a group of product alternatives customers consider during their choice procedure. Simonson [36] showed that choices are made in the context of a consideration set, i.e. a choice set. Since only differences in utility matter due to the nature of choice models, the selection of a product choice set exhibits great impact on customer choice. Methods for determining the appropriate choice set considering usage context are described in Section 4.

\section{Product design variables $-\mathbf{X}$}

Product design variables describe the engineering decisions involved in product design. In the jigsaw case, blade tooth height, stroke frequency, step distance between two teeth, etc., all belong to the product design variables $\mathbf{X}$.

\section{CHOICE MODELING FRAMEWORK IN USAGE CONTEXT-BASED DESIGN}

In order to capture the impact of usage context and utilize usage context information in a design process, a framework for choice modeling in UCBD is proposed. Our discussion follows the sequence of four major phases for implementing the choice modeling framework.

Phase I Collect usage context information and identify usage context attributes E. (usage context identification)

Phase II Collect customer choice data together with choice set information $J_{n}$, customer profile $\mathbf{S}$ and their usage context attributes $\mathbf{E}$. In a stated preference survey, a choice experiment representing different combinations of customer profile and usage context is designed where each respondent makes the selection among a 
choice set for given usage scenarios. For revealed preference data collection, all data from real customer purchases are recorded. (data collection)

Phase III Create a physics-based model or a human-appraisal-based ordered logit model for predicting engineering performance $\mathbf{Y}$ as a function of usage context attributes $\mathbf{E}$, customer profile $\mathbf{S}_{\mathbf{Y}}$, and design variables $\mathbf{X}$. (linking performance with usage context and customer profile)

Phase IV Create a choice model for market share and demand estimation (choice model estimation)

In the rest of this section, details for each phase are provided.

Phase I: Usage Context Identification

A successful product design requires an understanding of customers' needs so that the products produced will match customers' interest. Similarly, in the proposed choice modeling framework for UCBD (Figure 2), we start with a survey through which important usage context attributes and common usage context scenarios among target customers are identified. Widely used survey methodologies include focus groups, one-on-one interviews of experienced users, and observations of the product being used [30].

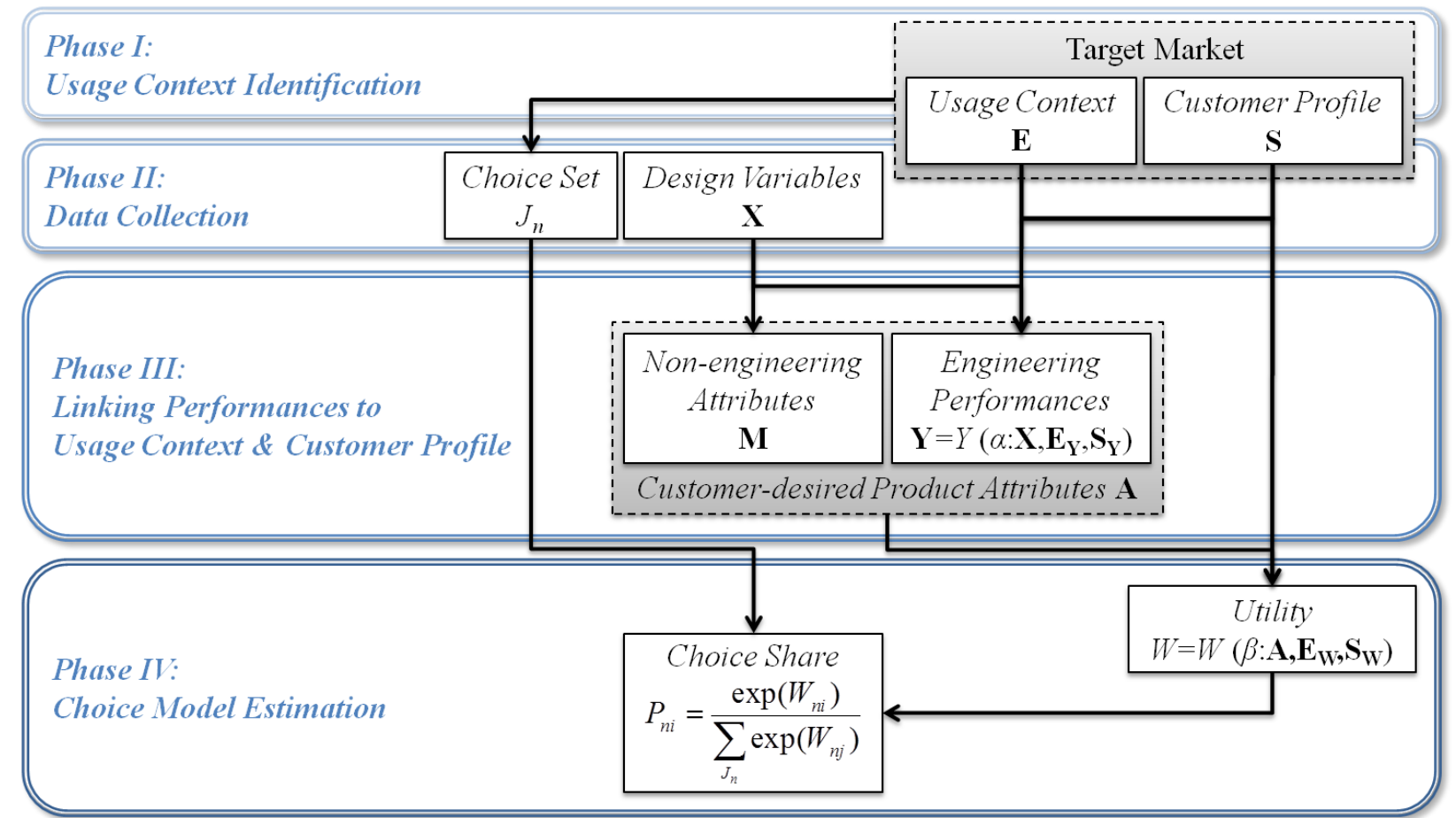

Figure 2: Choice Modeling for Usage Context-based Design

Following Belk's classification [25], usage context can be categorized into five types: physical surroundings, social surroundings, temporal perspective, task definition, and antecedent states. In Table 1, we use the jigsaw example to illustrate how the usage context attributes can be defined by following these five basic categories. It should be noted that based on Belk's classification, the scope of the usage context attributes is beyond the act of using the product, but also includes the context of purchase. 
He L., Chen W., Hoyle C., Yannou B., (2012), Choice modeling for usage context-based design. Journal of Mechanical Design, vol. 134(3), doi. DOI: 10.1115/1.4005860.

Table 1: Five Categories of Usage Context

\begin{tabular}{ll}
\hline \hline \multicolumn{1}{c}{ Usage Context Type } & \multicolumn{1}{c}{ Jigsaw Example } \\
\hline \multirow{3}{*}{ Physical surroundings } & Location of cutting, \\
& Accessibility of an outlet, \\
& Availability of workbench, \\
& Distance to do-it-yourself (DIY) stores. \\
\hline Social surroundings & Presence of children, neighbors. \\
\hline \multirow{4}{*}{ Temporal perspective } & Expected process duration, \\
& Lifetime of similar cutting tools in possession, \\
& Estimated time needed to purchase the tools in a \\
& nearby DIY store. \\
\hline & Material properties, \\
& Board thickness, \\
Task Definition & Minimal linear speed, \\
& Maximal vibration level, \\
& Noise and safety conditions, \\
& Accuracy requirements. \\
\hline \multirow{3}{*}{ Antecedent states } & Set of saw tools already in possession, \\
& New life conditions or projects, \\
& Cash at disposal. \\
\hline \hline
\end{tabular}

Physical surroundings are the most apparent characteristics of a usage. These characteristics include geographical location, weather condition, lighting, and other physical characteristics of a usage, as well as the distance to do-it-yourself (DIY) stores when the new tool is needed. In the case of using a jigsaw for cutting a board, the location where the operation must take place (indoor/outdoor), the accessibility of a power outlet, the availability of a workbench are typical examples of physical surroundings.

Social surroundings provide additional information about the social situation of a usage. Whether another person is present, his/her influence on the user, and other social characteristics belong to this category. For instance in cutting a board, one may prefer a jigsaw to a circular saw often used under these conditions, for reasons of safety and noise because of the presence of children nearby.

Temporal perspective refers to those aspects of the purchasing situation or to those of a given usage which are specific for a given range of time. For instance, the expected process (cutting task) duration may be a reason for preferring a circular saw to a jigsaw or a powerful jigsaw to a more basic one (faster linear speed). The age and expected lifetime of the cutting tools in possession are also deciding factors to determine how to upgrade the set of cutting tools in order to complete a set of cutting tasks. In terms of purchase situation, the time and emergency aspect for buying a new tool in a surrounding DIY store may also be determining under certain circumstances.

Task definition covers all features that explain the purpose of the purchase. For instance, one must consider the type of material to cut (wood, steel, etc.), the specification of the cut (blind or not, straight or wiggly, orthogonal or inclined), the properties of material (cutting hardness which is physically proportional to the material density), the thickness of the board to cut (beyond a certain thickness, the cut is impossible), the minimal linear speed that is acceptable when the 
user delivers the maximal amount of pushing arm forces and wrist torques, the maximal vibration level that is tolerable, or the admissible noise and minimal safety conditions.

Antecedent states define a dimension of usage which is antecedent to the purchase. The factors for a new jigsaw acquisition may be the set of saw tools one already possesses (circular, chain, panel, bow, miter, etc.) and their respective age and expected remaining lifetimes, a new life condition or project (moving from an apartment to a house, or a house remodeling), and the cash at one's disposal.

The above-mentioned five categories of usage context attributes can be used as a checklist in the process of determining the potential usage context attributes. For stated preference surveys, as will be demonstrated in the jigsaw case study (Section 5), a user survey is used to collect the set of primary usage context attributes E. For problems with a large number of usage context attributes $\mathbf{E}$, a cluster analysis becomes essential to reduce the possible combinations of usage context attributes $\mathbf{E}$ to a manageable size, and focus the study on a set of common usage context scenarios, i.e. the most common combinations of usage context attributes $\mathbf{E}$.

\section{Phase II: Data Collection}

Due to the nature of data collection, surveys can be divided into Stated Preference (SP) [37] and Revealed Preference (RP) [38]. SP refers to choice experiments where respondents are presented with a set of simulated product options from which they make a choice. This approach is attractive for model building because a high level of control can be exercised over the collected data, resulting in a data set optimized for choice modeling. However, SP data does not represent real purchase behavior and such surveys require significant time and additional cost to administer, thus resulting in a limited size and quality [39] dataset. For these reasons, it is sometimes desirable to utilize actual purchase data and customer satisfaction surveys collected.

In a stated preference survey, a choice experiment is conducted in which customers are asked to make a choice among several available alternatives under given usage context scenarios. Since the number of products available is usually much larger than the number of products a customer can use and compare in a choice experiment within a reasonable amount of time, an optimal experimental design can be applied to reduce the number of products in the choice set to a feasible level. For example, a nested design of experiments (DOE) on $\left(J_{n} \mid S, E\right)$ can be applied to find the optimal set of choice alternatives for respondents based on their customer profile $S$ and usage context $E$. The D-optimal experiment design algorithm for human appraisal surveys [40] can be used to select the products to include in the choice set for best model estimation.

A try-it-out survey is highly recommended for collecting SP data, in which customers are asked to use the products under given usage scenarios, rate the performances, and make a choice of one of the products. There are many advantages to conducting a try-it-out survey: first, handson experience is very important as it often simulates a real purchase process better for products that are typically tested prior to purchase; second, customers experience the product under certain usage contexts, which ensures the relevance; and third, assessments of the product performance reflect customers' perceived product performance. On the other hand, the try-it-out survey often requires more resources than a paper survey, where photos (or images) and data specification sheets are commonly used to present the products.

As for the revealed preference survey, all interesting product attributes, the customer profile, the usage context, and the choice set considered by each customer are recorded, together with the purchase decisions. RP data has the advantage in that it reflects consumers' real choice behavior; 
however, RP data may present issues with collinearity, endogeneity, and lack of randomization. An examination of the information matrix from choice model estimation could identify the possible orthogonality issues in RP data. In some cases, customers' ratings of product performances are also collected in RP surveys to capture customers' perceived product performances. Such level of detail is required to model the impact of usage context on product performance and customer preference as introduced next.

\section{Phase III: Linking Performance with Usage Context and Customer Profile}

This is a unique phase for UCBD applications in which product performances $\mathbf{Y}$ are formulated as a function of performance-related usage context attributes $\mathbf{E}_{\mathbf{Y}}$, performance-related customer profile attributes $\mathbf{S}_{\mathbf{Y}}$, and product design variables $\mathbf{X}$, as shown in Equation (1):

$$
\mathbf{Y}_{n i}=Y\left(\alpha: \mathbf{E}_{\mathbf{Y} n}, \mathbf{S}_{\mathbf{Y} n}, \mathbf{X}_{i}\right) .
$$

where the coefficients $\alpha$ can either be established by a physics-based model or determined through a human-appraisal-survey-based regression model. The physics-based model is constructed based on the physical relations. Taking the jigsaw design as an example, a system of equations can be derived to calculate the translational force and the torque on the user's wrist to assess the user's comfort level during the cutting process as a function of wood type and thickness as well as of admissible force and torque depending on the user experience [41]. The second approach utilizes rating data given by customers in a human appraisal survey and builds a regression model to predict the ratings of performances $\mathbf{Y}$. While the physics-based model saves the time and cost of a survey, a human appraisal survey can be used to assess either quantitative or qualitative performance perceived by the consumers. Such surveys can be integrated into the try-it-out survey for choice modeling, as described in Phase II. The ordered logit model [42] is used for modeling the discrete rating data in the hybrid electric vehicle case study in this work.

\section{Phase IV: Choice Model Estimation}

As shown in Figure 2, in Phase IV a predictive model of demand $Q$ is established using Discrete Choice Analysis (DCA), a statistical technique of building probabilistic choice models $[14,43]$. DCA is based upon the assumption that individuals seek to maximize their personal customer choice utility, $u$, when selecting a product from a choice set. The concept of choice utility is derived by assuming that the individual's $(n)$ true choice utility, $u$, for a design alternative, $i$, consists of an observed part $W$, and an unobserved random disturbance $\varepsilon$ (unobserved utility):

$$
u_{n i}=W_{n i}+\varepsilon_{n i} .
$$

As shown in Equation (3), the observed or deterministic part of utility $W_{n i}$ is expressed as a function of customer desired product attributes $\mathbf{A}$, usage context attributes $\mathbf{E}$, customer profile attributes $\mathbf{S}$.

$$
W_{n i}=W\left(\beta: \mathbf{A}_{n i}, \mathbf{E}_{\mathbf{W}_{n}}, \mathbf{S}_{\mathbf{W}_{n}}\right) .
$$

where $\mathbf{A}_{n i}$ denotes the customer-desired product attributes of respondent $n$, alternative $i, \mathbf{E}_{\mathbf{W} n}$ and $\mathbf{S}_{\mathbf{W} n}$ denotes the preference-related customer profile attributes and usage context attributes of respondent $n$. The coefficients $\beta$ are estimated based on the data collected in Phase III. From the 
observed utility, $W_{n i}$, the probability $P_{n i}$ of an individual $i$ choosing a given alternative $n$ can be estimated. By following the information flow in the four-phase diagram (Figure 2), we can see clearly how product design variables $\mathbf{X}$, together with the definitions of usage context $\mathbf{E}$ and consumer profile $\mathbf{S}$, are first mapped to product performance $\mathbf{Y}$ (Eqn. (1)), then to deterministic utility $W$ (Eqn.(2)), and finally to the probability of choice $P_{n i}$, which can be aggregated to the total market share based on predictions for a population. This flow creates a mathematical link between product design decisions, represented by $\mathbf{X}$, to consumer demand, represented by $P_{n i}$.

\section{JIGSAW EXAMPLE}

In this section, the design of a jigsaw is used to demonstrate the implementation of the proposed usage context-based choice modeling approach with stated preference data. The jigsaw is a common power tool for cutting wood. Under different usage contexts, the jigsaw performances, as well as the customers' preferences for the saw, change. The choice set considered in the user survey is formed by a few representative jigsaw products in the market. The four phases of choice modeling for UCBD are illustrated with the hypothetical saw design and a few representative attributes for demonstration. A choice model is built and estimated on synthetic survey data generated using a few key assumptions about customer preferences. Results are discussed which demonstrate the proposed framework.

- Phase I: Usage context identification

Phase I is completed with three tasks: collect usage information, identify common usage contexts through cluster analysis, and identify usages context attributes. We start with a user survey in which questions about primary usages are asked. It should be noted that the primary usage context is not limited to the most frequent usage context, and can be defined by the user. In some cases, for instance, a saw is expected to accommodate the most-demanding usage context. As described in Section 4, five categories of usage context can be used as a guideline for determining the usage context attributes. Figure 3 shows a small portion of the sample user survey questionnaire as an example. A few typical usage context questions for a jigsaw user would include wood type, working environment, etc.

Please answer the following question by choosing the best description of your primary saw usage context.

1. The woods you are cutting are: soft medium hard

2. The working environment of your cutting is: indoor outdoor

Figure 3: Sample User Survey Questionnaire for Phase I

In this case study, we select wood type and working environment as two usage context attributes $\mathbf{E}$ for demonstration purpose; wood type (it amounts to wood density in fact) is considered as a performance-related attribute $\mathbf{E}_{\mathbf{Y}}$ that influences product performance $\mathbf{Y}$, while both wood type and working environment are treated as preference-related attributes $\mathbf{E}_{\mathbf{W}}$ with an impact on customer preference. The wood type attribute is coded as 1 for soft, 2 for medium, and 3 for hard, while the working environment attribute is coded as 0 for indoor and 1 for outdoor. Based on the survey data of common usages, cluster analysis is performed. For our case study, indoor cutting for soft wood, outdoor cutting for medium wood, indoor cutting for medium wood, 
He L., Chen W., Hoyle C., Yannou B., (2012), Choice modeling for usage context-based design. Journal of Mechanical Design, vol. 134(3), doi. DOI: 10.1115/1.4005860.

and outdoor cutting for hard wood are identified to be the most common usages (Table 2) based upon the results from a k-means clustering analysis [44] on the hypothetical survey data with two usage context attributes, working environment and wood type, and $\mathrm{k}$ (number of clusters) $=3$.

Table 2: Common Usage Contexts Identified from Cluster Analysis

\begin{tabular}{cccl}
\hline \hline No. & Working environment & Wood type $\boldsymbol{E}_{\boldsymbol{2}}$ & \multicolumn{1}{c}{ Usage context description } \\
\hline 1 & $\boldsymbol{E}_{\boldsymbol{1}}$ & 1 & indoor cutting for soft wood \\
2 & 0 & 2 & outdoor cutting for medium wood \\
3 & 1 & 2 & indoor cutting for medium wood \\
4 & 0 & 3 & outdoor cutting for hard wood \\
\hline
\end{tabular}

- Phase II: Data collection

Various human-appraisal experiments can be utilized to collect consumers' preferences under different usage contexts as described in Section 4. The question lies in how to minimize the amount of surveys to cover the various attributes included in choice modeling. Here we assume that each respondent is surveyed for more than one usage context (but only one primary usage context at a time in the choice experiment). We also assume that all respondents have some level of experience with the product and are able to differentiate between the different usage contexts described in the survey questionnaire. Eight jigsaw products available in the market are considered, but only four products that are most relevant for a given usage context form the choice set $J_{n}$ in each choice experiment. Three customer profile attributes are included: gender $(0$ for male and 1 for female), income (annual income in \$1,000s), and skill level (1 for elementary user, 2 for experienced user, and 3 for professional user).

The synthetic data are simulated with 500 respondents, 4 choices alternatives, and 4 usage contexts $(8,000$ observations in total) based on a few key assumptions about customer preferences. Table 3 presents three categories of attributes considered for choice modeling, including three customer-desired product attributes $\boldsymbol{A}$ (price, advance speed, and comfort), two usage context attributes $\mathbf{E}$ (working environment, and cutting board wood type), three customer profile attributes $\mathbf{S}$ (income, gender, and skill level), together with four design variables $\mathbf{X}$ (blade tooth height, stroke frequency, blade translation, and step distance between teeth).

Table 3: List of Attributes and Design Variables included in Jigsaw Case Study

\begin{tabular}{|c|c|c|}
\hline \multicolumn{3}{|c|}{ Customer Desired Product Attributes A } \\
\hline$M$ & Price & \\
\hline$Y_{t 1}$ & Advance speed $S_{a}$ & \\
\hline$Y_{t 2}$ & Comfort level $P_{\text {comfort }}$ & $\%$ \\
\hline \multicolumn{3}{|c|}{ Usage Context Attributes $\mathbf{E}$} \\
\hline$E_{1}$ & Working environment & indoor, outdoor \\
\hline$E_{2}$ & Cutting board wood type & soft, medium, hard \\
\hline \multicolumn{3}{|c|}{ Customer Profile $\mathbf{S}$} \\
\hline$S_{1}$ & Income & $\begin{array}{l}\text { uniform dist. , [50k, } \\
150 \mathrm{k}]\end{array}$ \\
\hline$S_{2}$ & Gender & male, female \\
\hline$S_{3}$ & Skill level & $1,2,3$ \\
\hline \multicolumn{3}{|c|}{ Product Design Variables $\mathrm{X}$} \\
\hline
\end{tabular}


He L., Chen W., Hoyle C., Yannou B., (2012), Choice modeling for usage context-based design. Journal of Mechanical Design, vol. 134(3), doi. DOI: 10.1115/1.4005860.

\begin{tabular}{ll}
$F$ & Stroke frequency \\
$A$ & Blade translation \\
$S$ & Step distance between teeth \\
\hline
\end{tabular}

It should be pointed out that the experimental design is not unique, and can be designed based on the number of respondents who are available [40,45]. For example, when there are a large number of respondents, fewer choice experiments can be used for each respondent, than in experiments with fewer respondents. Pairing the usage contexts to customers' primary usages is recommended, as it yields a better understanding of the influence of usage context attributes. If a two-stage (consumer) decision making process is considered (i.e., first the choice set is selected followed by the specific product), the survey will be designed for predicting the choice set for each customer first.

- Phase III: Linking performances with usage context and customer profile

In this study, the link between product variables $\mathbf{X}$, performance-related usage context attributes $\mathbf{E}_{\mathbf{Y}}$, performance-related customer profile attributes $\mathbf{S}_{\mathbf{Y}}$, and engineering performance $\mathbf{Y}$ (Eqn. (1)), is established using a series of physics-based equations based on the functional principles of the jigsaw [41, 46]. Both engineering performances $\mathbf{Y}$ considered in this study, the advance speed $S_{a}$ and comfort level $P_{\text {comfort }}$, belong to $Y_{t}$ (performance of transformation). The advance speed $S_{a}$ is calculated as follows:

$$
S_{a}=\frac{2 H_{d} f \cdot A}{s}
$$

where $H_{d}$ is the blade tooth height, $f$ is the stroke frequency, $A$ is the blade translation, and $s$ is the step distance between two teeth. All variables in the equation $\left(H_{d}, f, A\right.$ and $\left.s\right)$ are product design variables $\mathbf{X}$; usage context doesn't influence this particular performance. The comfort level $P_{\text {confort }}$ is associated with the required wrist torque with respect to user' maximum wrist capability, as shown in the following equation:

$$
P_{\text {comfort }}=1-\left|\frac{M_{w}}{M_{w-\max }}\right|
$$

where $M_{w}$ is the wrist torque and $M_{w-\max }$ is the maximal wrist torque that can be delivered by the user. In [41, 46], $M_{w}$ is modeled as a function of both product design variables $\mathbf{X}$ and usage context attributes $\mathbf{E}_{\mathbf{Y}}$ (i.e., wood type), while $M_{w-\max }$ is modeled as a function that depends on customer profile attributes $\mathbf{S}_{\mathbf{Y}}$. Details of the above physics-based equations can be found in references $[41,46]$.

- Phase IV: Choice model estimation

A multinomial logit model is estimated using STATA [47]. The goodness of fit, measured by the rho square is 0.82 with a log likelihood of -500.76 . The coefficients the estimators, standard errors, and the significance of their $p$ values are provided in Table 4 . The price $M$ is divided by the income $S_{1}$, as customers with higher income are expected to be less sensitive to the price. The sign of the $M / S_{1}$ coefficient shows that price has a negative impact on the utility function. The coefficients of $Y_{t l}$ and $Y_{t 2}$, are both significant, showing that both performances are important in 
users' choice. Therefore, in-service and service performance results must be considered in the jigsaw design. The coefficient for $S_{2} * Y_{t 2}$ is significantly positive, which indicates that the female users tend to care more about the comfort than male users do. This is important to consider in the design process if the intended market for the saw has a sizable female population. Similarly, the coefficient for $S_{3} * Y_{t l}$ is significantly positive, meaning that skilled users care more about the advance speed during cutting, compared with amateur users. As for the interactions between performance $Y_{t l}$ and usage context variable $E_{1}$ (indoor / outdoor) and performance $Y_{t 2}$ and usage context variable $E_{2}$ (wood type), both coefficients are statistically significant, which indicates that both $E_{1}$ and $E_{2}$ belong to the category of preference-related usage context variable $E_{W}$. Moreover, the negative sign suggests that $Y_{t 2}$ (comfort) is less important when users are cutting outdoors $\left(E_{l}=1\right)$, while the positive sign indicates that advance speed is more critical when users are cutting hard wood $\left(E_{2}=3\right)$. This again provides direction in the design process: for example, if the intended market for the saw is users cutting soft to medium woods (e.g. framing materials), then advance speed is not as important in the design than if the intended market is for those cutting hard woods (e.g. hardwood flooring). The results from this case study are consistent with the general trend in customer preferences assumed in data generation.

Table 4: Multinomial Logit Model Estimation Results in Jigsaw Case Study

\begin{tabular}{lrrr}
\hline \hline Variables & Coef. & Std.Err. & P>|z| \\
\hline$Y_{t 1}$ & 5.39 & 1.42 & 0.00 \\
$Y_{t 2}$ & 27.30 & 1.98 & 0.00 \\
$M / S_{1}$ & -35.86 & 1.76 & 0.00 \\
$S_{2} * Y_{t 2}$ & 4.13 & 1.51 & 0.01 \\
$S_{3} * Y_{t 1}$ & 7.42 & 0.49 & 0.00 \\
$E_{1} * Y_{t 2}$ & -4.94 & 1.62 & 0.00 \\
$E_{2} * Y_{t 1}$ & 4.06 & 0.49 & 0.00 \\
\hline \hline
\end{tabular}

\section{HYBRID ELECTRIC VEHICLE EXAMPLE}

Alternative fuel vehicles have drawn increasing attention in the past few years, because of their potential to reduce greenhouse-gas emissions and utilize renewable energy sources [48-50]. However, understanding consumer choices of alternative fuel vehicles is challenging because their preference construction process involves many aspects beyond traditional engineering considerations, which calls for a comprehensive modeling framework to incorporate usage context into engineering design. Taking hybrid electric vehicles (HEVs) as an example, vehicle performances, such as mileage per gallon, often depend highly on their usage contexts, while consumers" attitudes towards new technology, especially "green" products, are strongly influenced by their intended usage. In this section, a hybrid electric vehicle case study is used to illustrate the proposed usage context based choice modeling framework. Different from the jigsaw problem, the revealed preference data collected by JD Power and Associates for both HEVs and conventional vehicles (CVs) is utilized for model estimation. It should be noted that in our current study, the impact of HEV policies and other purchase incentives is not considered.

- Phase I: Usage context identification

Two usage context attributes are considered for HEV choice modeling: a local/highway indicator and average miles driven daily. While both attributes are treated as preference-related 
attributes $\mathbf{E}_{\mathbf{W}}$, the local/highway indicator is also considered as a performance-related attribute $\mathbf{E}_{\mathbf{Y}}$ in mileage per gallon (MPG) calculation, as detailed later. The local/highway indicator is assessed based on the combined MPG published by US Environmental Protection Agency [51] and the estimated MPG given by survey respondents. The indicator is a continuous parameter, ranging from 0 for pure local driving to 1 for pure highway driving. It is assumed that the local/highway indicator reflects the general driving condition a respondent faces, therefore the vehicle usage context. The local/highway driving condition not only greatly impacts vehicles' performances, e.g. MPG, but is also expected to influence customers' choice preference for hybrid vehicles. The other usage context attribute considered is average miles driven daily, a commonly used descriptor of customers' travel pattern. The data is derived from the recorded miles driven in the first three months in the J.D. Power and Associates data. This is an important usage context attribute in designing new HEV and future plug-in hybrid electric vehicles (PHEV), as average miles driven daily strongly influence the target performance of the batteries.

- Phase II: Data collection

The Vehicle Quality Survey (VQS) conducted by J.D. Power and Associates belongs to the revealed preference data because the customer satisfaction survey is strictly about the new vehicles respondents purchased instead of hypothetical design alternatives. In the $2007 \mathrm{VQS}$, vehicle purchase data from 90,000 nation-wide respondents on over 300 vehicles in the market are collected, including data for 11 HEV models. Further, respondents' demographic attributes and their usage patterns are recorded in the questionnaire. For model estimation, data collected from 8025 respondents, who reported their vehicle choice sets, are selected. The attributes and design variables included in the choice model are summarized in Table 5.

Table 5: List of Attributes and Design Variables included in HEV Case Study

\begin{tabular}{lll}
\hline \hline \multicolumn{2}{l}{ Customer-desired product attributes A } & \\
\hline$A_{1}$ & Price & Price paid, excluding tax, license, trade-in, etc. \\
$A_{2}$ & MPG & Mileage Per Gallon under usage \\
$A_{3}$ & Vehicle origin & Domestic / European / Japanese / Korean \\
$A_{4}$ & Vehicle size & Compact / Midsize / Large / Premium \\
$A_{5}$ & Vehicle type & Mini / Car / SUV / Minivan / VAN / MAV / Pickup \\
$A_{6}$ & Hybrid electric vehicle & 1 for hybrid, 0 for conventional \\
$A_{\text {exterior }}$ & Exterior attractiveness rating & \\
$A_{\text {interior }}$ & Interior attractiveness rating & \\
$A_{\text {storage }}$ & Storage and space usage rating & \\
$A_{\text {audio }}$ & Audio rating & \\
$A_{\text {seats }}$ & Seats rating & Discrete rating on a scale from 1 to 10 \\
$A_{\text {hvac }}$ & HVAC rating & \\
$A_{\text {dynamics }}$ & Driving dynamics rating & \\
$A_{\text {engine }}$ & Engine and transmission rating & \\
$A_{\text {safety }}$ & Visibility and safety rating & \\
\hline \hline Usage context attributes $\mathbf{E}$ & \\
\hline$E_{1}$ & Local / highway indicator & $0-$ local, 1 - highway \\
$E_{2}$ & Average miles driven daily & Unit: miles \\
\hline Customer profile attributes $\mathbf{S}$ & \\
\hline$S_{1}$ Gender & 1 for male, 2 for female \\
$S_{2}$ & Age & Age \\
$S_{3}$ & Income \\
\hline
\end{tabular}


He L., Chen W., Hoyle C., Yannou B., (2012), Choice modeling for usage context-based design. Journal of Mechanical Design, vol. 134(3), doi. DOI: 10.1115/1.4005860.

\begin{tabular}{lll}
\hline \hline$S_{4}$ & Children & Number of children under 20 living in the household \\
$S_{5}$ & Education & Level of education completed \\
\hline \hline \multicolumn{1}{l}{ Product design variables $\mathbf{X}$} & \\
\hline$X_{1}$ & Exterior dimensions & Vehicle length / width / height (unit: in.) \\
$X_{2}$ & Vehicle weight & Unit: lbs \\
$X_{3}$ & Interior dimensions & Front head / shoulder / hip / leg room (unit: in.) \\
& & Rear head / shoulder / hip / leg room (unit: in.) \\
$X_{4}$ & Storage capacity & Luggage capacity \\
& & Cargo capacity \\
$X_{5}$ & Engine specifications & Engine size \\
& & Number of cylinders \\
$X_{6}$ & Performance & Horsepower \\
$X_{7}$ & MPG targets & Torque \\
\hline \hline
\end{tabular}

There are 288 car models covered in the data set, each of them is chosen by at least one respondent. Fifteen customer-desired product attributes $\mathbf{A}$ are selected including price, vehicle origin, vehicle size, vehicle type, mileage per gallon (MPG), hybrid electric vehicle indicator, and nine rating scores given by the respondents. The attribute "price" is the money respondents paid excluding tax, license, trade-in and etc. Since VQS only provides price for the purchased vehicles, the price data for other vehicles considered are estimated from a linear regression model based on vehicle make and model, and customers' geographic locations. As shown in the third column in Table 5, vehicle origins are categorized as domestic, European, Japanese, and Korean; vehicle sizes are grouped into compact, midsize, large, and premium; vehicle type includes mini, car, sport utility vehicles (SUV), minivan, van, multi-activity vehicles (MAV), and pickup. The hybrid electric vehicle indicator, coded as 1 for hybrid vehicles, and 0 for conventional vehicles, reflects customers' attitude toward new hybrid technology. Nine aspects of a vehicle, including exterior attractiveness, interior attractiveness (as stated by the average purchaser), storage and space usage, audio/entertainment/navigation system, seats, heating ventilation and air conditioning, driving dynamics, engine and transmission, and visibility and driving safety, are rated on a scale of 1 to 10,10 being the most satisfactory. These discrete ratings are included in the choice modeling procedure, as they are considered to be a good measure of customers' perceived vehicle performance (quality).

Meanwhile, gender, age, household income, number of children under age 20 living together and education level, are included as five customer profile attributes $\mathbf{S}$. Among the set of $\mathbf{S}$, critical preference-related attributes $\mathbf{S}_{\mathbf{W}}$ will be identified through choice modeling in Phase IV. All five $\mathbf{S}$ attributes are considered in the ordered logit regression for predicting the performance rating scores, as will be shown in Phase III.

- Phase III: Linking performances with usage contexts and consumer profile

Different from the jigsaw example in which physics-based modeling can be used to establish the relationship between performance and usage context attributes, in the HEV example, respondent survey data is used to create the relationship as shown in Equation (1) by using the ordered logit modeling method [18] for nine customer desired product attributes (A) in the form of ratings. Here the ratings are used to represent product performances $\mathbf{Y}$. Seven high level engineering design variables $\mathbf{X}$ are used in this case study, including exterior dimension, interior dimension, performance, MPG targets, etc. The obtained ordered logit models are also used to predict the 
He L., Chen W., Hoyle C., Yannou B., (2012), Choice modeling for usage context-based design. Journal of Mechanical Design, vol. 134(3), doi. DOI: 10.1115/1.4005860.

ratings of other vehicle designs in the choice set as customers only rate the vehicles they purchase. This limitation of the rating data in VQS may cause ownership bias in model estimation and potentially lead to inaccurate estimates of some coefficients due to the missing heterogeneity in owners' ratings. Further details for implementing the ordered logit model based on the VQS data by JD Power and Associates can be found in [52]. In addition to the design variables $\mathbf{X}$, customer profile $\mathbf{S}_{\mathbf{Y}}$ such as gender, age, etc., are included to capture customers' heterogeneity in rating. The coefficients estimators are later used for what-if-scenario analysis to forecast potential market share for targeting customer and usage attributes.

Furthermore, the impact of usage context (local/highway indicator $E_{l}$ ) on the vehicle performance $\left(A_{2}\right.$, mileage per gallon) is represented in the following equation:

$$
A_{2}=\frac{1}{\frac{1-E_{1}}{M P G_{\text {city }}}+\frac{E_{1}}{M P G_{\text {highway }}}} .
$$

where $M P G_{\text {city }}$ and $M P G_{\text {highway }}$ belong to the product design variables $\mathbf{X}$ listed in Table 5.

- Phase IV: Choice model estimation

In Phase IV, as a result of choice modeling, interactions between customer-desired product attributes $\mathbf{A}$, usage context attributes $\mathbf{E}$, and customer demographics $\mathbf{S}$ are explicitly modeled in the utility function. The coefficients for all attributes and their interactions based on a multinomial logit model estimation (MNL with $\mathbf{E}$ ) are listed in Table 6, together with the estimation results from a multinomial logit model without usage context attributes (MNL without E) as a comparison.

Table 6: Selected Coefficients of MNL with E and MNL without E for HEV

\begin{tabular}{|c|c|c|c|c|c|c|}
\hline \multirow[b]{2}{*}{ Attributes } & \multicolumn{3}{|c|}{ MNL without $\mathrm{E}$} & \multicolumn{3}{|c|}{ MNL with E } \\
\hline & Coefficients & Std.Err. & Sig. & Coefficients & Std.Err. & Sig. \\
\hline$A_{1} / S_{3}$ & -0.0003 & 0.0000 & $* * *$ & -0.0004 & 0.0000 & $* * *$ \\
\hline$A_{2}$ & I & I & & -3.1080 & 0.0846 & $* * *$ \\
\hline$A_{2 \_}$city & 0.0456 & 0.0139 & $* * *$ & / & I & \\
\hline$A_{2 \_}$highway & -0.0791 & 0.0141 & $* * *$ & I & I & \\
\hline$E_{1} * A_{2}$ & I & I & & 5.9454 & 0.1697 & $* * *$ \\
\hline$E_{2} * A_{2}$ & I & I & & 0.0002 & 0.0003 & \\
\hline$A_{3 \_}$European & 1.9353 & 0.0900 & $* * *$ & 2.1886 & 0.1045 & $* * *$ \\
\hline$A_{3 \_J a p a n e s e}$ & 0.2314 & 0.0505 & $* * *$ & 0.5161 & 0.0576 & $* * *$ \\
\hline$A_{3 \_}$Korean & 1.2617 & 0.0904 & $* * *$ & 1.3313 & 0.1065 & $* * *$ \\
\hline$A_{4}$ Large & -0.5636 & 0.0907 & $* * *$ & -0.9111 & 0.1024 & $* * *$ \\
\hline$A_{4 \_}$Medium & 0.0907 & 0.0534 & $* *$ & -0.1376 & 0.0601 & $*$ \\
\hline$A_{4} \_$Premium & -0.2496 & 0.0780 & $* * *$ & -0.3136 & 0.0888 & $* * *$ \\
\hline$A_{5} \_M A V$ & -0.9746 & 0.0782 & $* * *$ & -1.3558 & 0.0847 & $* * *$ \\
\hline$A_{5 \_}$Mini & 0.6717 & 0.1312 & $* * *$ & 1.3347 & 0.1568 & $* * *$ \\
\hline$A_{5 \_}$Minivan & -0.6733 & 0.1393 & $* * *$ & -0.9826 & 0.1607 & $* * *$ \\
\hline$A_{5 \_}$Pickup & 1.6354 & 0.1973 & $* * *$ & 1.4446 & 0.2227 & $* * *$ \\
\hline$A_{5 \_} S U V$ & -0.0632 & 0.0971 & & -0.2733 & 0.1115 & $*$ \\
\hline$A_{5-} \operatorname{Van}$ & 1.2458 & 1.2891 & & 0.8806 & 1.3755 & \\
\hline
\end{tabular}


He L., Chen W., Hoyle C., Yannou B., (2012), Choice modeling for usage context-based design. Journal of Mechanical Design, vol. 134(3), doi. DOI: 10.1115/1.4005860.

\begin{tabular}{lrrrrrc}
\hline \hline $\boldsymbol{A}_{\boldsymbol{6}} \boldsymbol{d}$ _hybrid & 2.8933 & 0.5878 & $*$ & 57.0667 & 2.4840 & $* * *$ \\
$\boldsymbol{E}_{\boldsymbol{I}} * \boldsymbol{A}_{\boldsymbol{6}} \boldsymbol{d}$ _hybrid & $/$ & $/$ & & -105.8431 & 4.8316 & $* * *$ \\
$\boldsymbol{S}_{5}{ }^{*} \boldsymbol{A}_{\boldsymbol{6}} \boldsymbol{d}$ _hybrid & 0.2875 & 0.1213 & $* * *$ & 0.1686 & 0.0846 & $*$ \\
$\boldsymbol{A}_{\text {exterior }}$ & 0.0593 & 0.0340 & $* * *$ & 0.0407 & 0.0385 & \\
$\boldsymbol{A}_{\text {interior }}$ & 0.4835 & 0.0174 & $* * *$ & 0.4585 & 0.0190 & $* * *$ \\
$\boldsymbol{A}_{\text {storage }}$ & 0.5804 & 0.0146 & $* * *$ & 0.6253 & 0.0175 & $* * *$ \\
$\boldsymbol{A}_{\text {audio }}$ & 0.1741 & 0.0312 & $* * *$ & 0.1421 & 0.0355 & $* * *$ \\
$\boldsymbol{A}_{\text {seats }}$ & 0.1636 & 0.0373 & $* * *$ & 0.1046 & 0.0424 & $*$ \\
$\boldsymbol{A}_{\boldsymbol{H} \text { VAC }}$ & 0.1242 & 0.0340 & $* * *$ & 0.1285 & 0.0391 & $* * *$ \\
$\boldsymbol{A}_{\text {dynamics }}$ & 0.2362 & 0.0380 & $* * *$ & 0.1640 & 0.0433 & $* * *$ \\
$\boldsymbol{A}_{\text {engine }}$ & 0.2565 & 0.0301 & $* * *$ & 0.3061 & 0.0343 & $* * *$ \\
$\boldsymbol{A}_{\text {safety }}$ & *.0999 & 0.0384 & $* *$ & 0.0455 & 0.0437 & \\
\hline \hline & $*$ Significant with p value $<=\mathbf{0 . 0 0 1 .}$ & &
\end{tabular}

From the results of the MNL including $\mathbf{E}$ attributes in modeling, we note that the coefficient for price/income is negative as expected. Only two customer profile attributes, household income and education level, are statistically significant as preference-related attributes $\mathbf{S}_{\mathbf{W}}$. A positive estimator for $E_{1} * A_{2}$ indicates that the usage context attribute $E_{1}$ (local/highway indicator) has a positive impact on customers' preference on MPG measure. In other words, people primarily driving on highways tend to care more about the MPG value, in addition to the utility increase experienced by the change in MPG. Moreover, the attitude toward HEV itself has a fairly large coefficient estimator of 57.0667, which shows that people driving locally tend to favor HEV. Similarly as we expected, highway drivers do not prefer HEVs, as shown in the negative coefficient estimator of the $E_{1}$ and HEV indicator interaction $\left(E_{1} * A_{6}\right)$. This finding presents an opportunity to design a HEV which performs well in highway driving to help overcome this issue. On the other hand, most coefficients from the MNL without modeling $\mathbf{E}$ have the same sign as the ones in MNL with $\mathbf{E}$, but they are very different in magnitude, as the usage heterogeneity is not explicitly modeled. Inclusion of usage context will help designers more specifically target the vehicle design to the usage contexts of the intended market for the vehicle. It should be noted that the results shown in Table 6 are attained through sequential estimation of a hierarchical model and do not account for error propagation. Moreover, the negative coefficient of $A_{2}$ highway is due to high correlation between $A_{2 \_}$city and $A_{2}$ highway. Multicolinearity between explanatory variables should be cautioned in model estimation.

Goodness-of-fit Measures

Goodness-of-fit measures based upon the log-likelihood of the converged model, such as the likelihood ratio index $\rho^{2}$ (also known as pseudo R-square), reflect how well the estimated model predicts actual individual choices in the data set. Higher values of $\rho^{2}$ indicate better predictions of the choices. As shown in Table 7, a significantly higher log-likelihood of -4825.26 and subsequently $\rho^{2}$ value of 0.5663 are achieved using the MNL model with usage context attributes $\mathbf{E}$ versus the MNL model without $\mathbf{E}$. This implies that introducing the usage context attributes in choice modeling has captured the systematic taste heterogeneity of customers under different usage contexts. This is important for designers so that they may best understand the preferences and usage contexts of the intended users. 
He L., Chen W., Hoyle C., Yannou B., (2012), Choice modeling for usage context-based design. Journal of Mechanical Design, vol. 134(3), doi. DOI: 10.1115/1.4005860.

Table 7: Model Statistics of MNL without E and with E

\begin{tabular}{lrr}
\hline Multinomial Logit Model & without E & with E \\
\hline Log likelihood at zero & -11125.01 & -11125.01 \\
Log likelihood at convergence & -6178.62 & -4825.26 \\
$\rho^{2}$ & 0.4203 & 0.5663 \\
\hline \hline
\end{tabular}

\section{Cross-validation}

For cross-validation of a choice model, the original data are divided into 5 subsets of samples. For each of the five cross-validation tests, a choice model is trained on 4 subset samples and later validated using the remaining hold-out sample. The likelihood ratio index $\rho^{2}$ values and hit rates (percentage of correctly predicted choices) are calculated and averaged out, as listed in Table 8 .

Table 8: 5-fold Cross-Validation Results for UCBCM of HEVs

\begin{tabular}{lrrrr}
\hline \multicolumn{1}{c}{ Test } & \multicolumn{2}{c}{ MNL without E } & \multicolumn{2}{c}{ MNL with E } \\
\hline Measure & $\rho^{2}$ & hit rate & $\rho^{2}$ & hit rate \\
\hline 1 & $58.72 \%$ & $67.41 \%$ & $68.10 \%$ & $75.76 \%$ \\
2 & $57.10 \%$ & $66.60 \%$ & $66.87 \%$ & $76.20 \%$ \\
3 & $56.10 \%$ & $66.42 \%$ & $65.61 \%$ & $74.02 \%$ \\
4 & $56.35 \%$ & $65.67 \%$ & $65.84 \%$ & $74.14 \%$ \\
5 & $55.77 \%$ & $66.79 \%$ & $65.98 \%$ & $75.20 \%$ \\
Average & $56.81 \%$ & $66.58 \%$ & $66.48 \%$ & $75.07 \%$ \\
\hline \hline
\end{tabular}

On average, the likelihood ratio index $\rho^{2}$ shows an over $17 \%$ improvement from $56.81 \%$ in the MNL without $\mathbf{E}$ to $66.48 \%$ in the MNL with $\mathbf{E}$. The hit rate, though not theoretically consistent with random utility theory, is another commonly used measure of the prediction accuracy of an estimated model at the individual level. It is calculated by dividing the number of correctly predicted choices by the total number of respondents. Similar to $\rho^{2}$, the hit rate increases from $66.85 \%$ in the MNL without $\mathbf{E}$ to $75.07 \%$, which shows that usage context greatly influences customers' choice and should be modeled explicitly. As the choice model estimation and cross-validation are performed with choice set information from the VQS, future work is needed to address the challenges in predicting choice set construction for new market.

\section{What-If-Scenario Analysis}

With the formulations described above and choice model results from MNL with E, a prediction model can be built to forecast the customers' choice. For example, a target population of 260 customers is simulated with customer profile distribution drawn from the hybrid owner pool in VQS 2007 data set. Assuming that they are choosing a new vehicle to purchase from a choice set of 4 car models selected from 10 car models available in the market. The ten car models, among which two (vehicle 4 and vehicle 8) are HEVs, are selected based on their popularity in the choice set of customers who considered at least one HEV. The choice set of each customer can also be predicted using statistical learning or data mining methods with existing market data. Since not all customers would consider a hybrid electric vehicle when they shop for a new car, we assume that $40 \%$ of customer would include hybrid electric vehicles in their choice set, while the rest of them don't. Additionally, we consider a series of nine different usage contexts: a uniformly distributed local/highway indicator with 0.2 range and mean value 
from 0.1 to 0.9 (with 0.1 interval), while average miles driven daily matches with the original dataset. Aggregated choice probability in target population calculated using our proposed framework is summarized in Figure 4.

In Figure 4, the grey lines (conventional vehicles) and green lines (hybrid electric vehicles) on the left hand side show the predicted choice probability by MNL with $\mathbf{E}$, while the red lines on the right hand side represent the constant choice probability predicted by MNL without $\mathbf{E}$. For instance, when the target population, on average, drives $40 \%$ under local conditions, the hybrid electric vehicle 4 and vehicle 8 have the predicted choice probabilities of $10.72 \%$ and $12.64 \%$, respectively in MNL with $\mathbf{E}$, as opposed to the constant $7.32 \%$ and $8.11 \%$ in MNL without $\mathbf{E}$. According to the prediction from the MNL with $\mathbf{E}$, their predicted market shares gradually decrease, as $\mathrm{E} 1$ increases. When $\mathrm{E}_{1}$ is less than or equal to 0.3 , conventional vehicle 1 have the largest market share, closely followed by conventional vehicle 5. When $\mathrm{E}_{1}$ increases to 0.5 , the predicted choice probabilities change significantly, as shown in the middle of the figure. Each car model has its niche in the market. Similarly, when $\mathrm{E}_{1}$ is larger than or equal to 0.6, conventional vehicle 9 becomes the dominant car model, as it has the highest choice probability. This suggests that customers with extreme driving conditions ( $E_{1}$ close to 0 or 1$)$ have stronger, or clearer preferences for a specific car model, which is consistent with our experience. In comparison, the predicted dominant vehicle choice by the MNL without $\mathbf{E}$ turns out to be conventional vehicle 2 with a choice probability of $16.51 \%$, which is significantly different from the one predicted by the MNL with $\mathbf{E}$. Since the missing usage information plays a key role in customer choice and it is natural to expect that customers make distinctive decisions when usage context changes, the MNL with $\mathbf{E}$ is able to reveal relationships between usage context and consumer preference for product attributes that would not be revealed without E. Accurately predicting the choice probabilities (i.e. market share) for a given vehicle design, consumer population and set of usages is an important tool for vehicle designers to tailor the vehicle design to the target market as closely as possible. Further research is needed to assess implications of model specification assumptions and to test external validity on predictions of choice shares for different usage contexts.

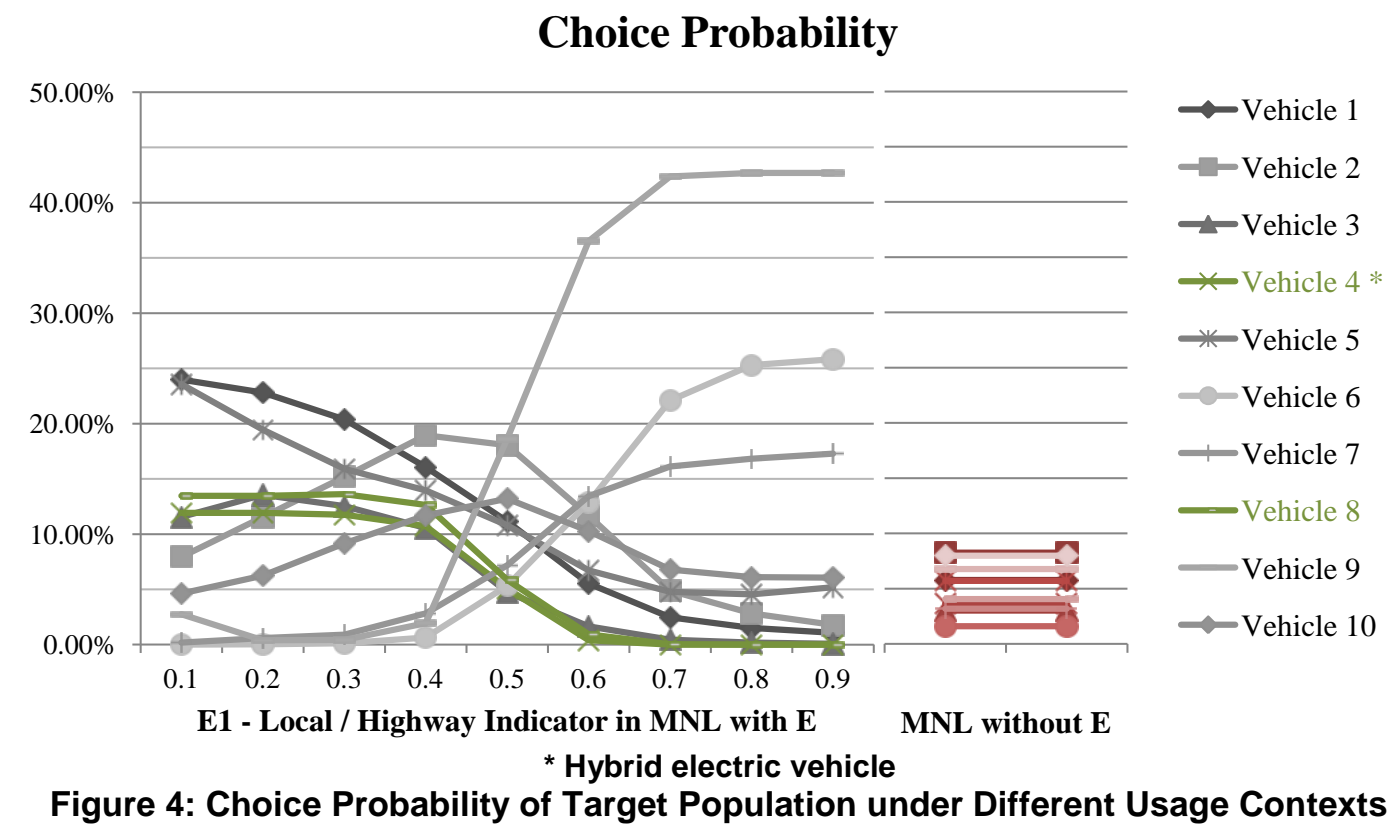


He L., Chen W., Hoyle C., Yannou B., (2012), Choice modeling for usage context-based design. Journal of Mechanical Design, vol. 134(3), doi. DOI: 10.1115/1.4005860.

\section{CONCLUSION}

In this work, we propose a choice modeling framework for Usage Context-based Design (UCBD) to quantify the impact of usage context on customer choices. Previous works have illustrated the importance of considering usage context in design, but did not present a systematic and quantitative approach to choice modeling. The primary focus of this work is the development of a systematic UCBD taxonomy and a step-by-step procedure to quantitatively assess the impact of usage context on product performance and consumer preferences.

A taxonomy for UCBD is first defined by following the established classification in the market research domain and the needs associated with choice modeling. The step-by-step procedure for creating choice models in UCBD is then presented. To facilitate the identification of usage contexts in Phase I, it is recommended to elicit the usage context attributes from five categories of product usages including physical surroundings, social surroundings, temporal perspective, task definition, and antecedent states. In Phase II data collection, both the methods of Stated Preference and Revealed Preference surveys are presented to account for the choices respondents make conditional on the given usage context, which allows us to examine simultaneously the influence of product design, customer profile, usage context, and their interactions, on consumer choices. Furthermore, Phase III is a unique step in a quantitative usage context-based design process in which the influence of usage context and consumer profile on product performance is analytically modeled. Additionally, in Phase IV, usage context enters into an individual's choice utility function directly to capture its influence on product preferences. In Phases III and IV of modeling, both customer profile attributes $\mathbf{S}$ and usage context attributes $\mathbf{E}$ are further classified into performance-related $\mathbf{S}_{\mathbf{Y}}, \mathbf{E}_{\mathbf{Y}}$ and preference-related $\mathbf{S}_{\mathbf{W}}$ and $\mathbf{E}_{\mathbf{Y}}$ to differentiate their impact on product performance and customer preferences, respectively. The usage context choice modeling approach in this work represents a significant expansion of traditional choice modeling approaches in the design literature.

Two case studies, a jigsaw design example with synthetic stated preference data and a hybrid electric vehicle example with real revealed preference data, illustrate the proposed modeling framework. Both case studies follow the four-phase modeling procedure. The jigsaw case study emphasizes usage context identification, data collection with stated preference surveys, and the use of physics-based modeling to capture the impact of usage context on performance. On the other hand, more details of the modeling steps (Phases III and IV) are reported in the hybrid electric vehicle case study based on its revealed preference survey data that reflects customers' real choices and the use of ordered-logit modeling for predicting customer ratings for system attributes. Results from both examples demonstrate the impact of usage context upon customer preference as well as product performance. A set of validation tests are included for the HEV case study which demonstrate the necessity of expanding a traditional choice modeling framework to include usage context for improved model predictive capability. What-if-scenario analysis in the hybrid electric vehicle example showed that predicted choice share in the target market changes in response to the change of performance ratings in distinctive usage contexts for given vehicle designs, which illustrates the potential of the proposed choice modeling framework in supporting engineering product design. An optimization problem can be formulated using the proposed framework to determine the optimal performance targets for engineering design. For example, in the case of HEV battery design, performance targets include both city and highway MPG as well as vehicle horsepower and torque. This work is limited to single primary usage of a product which may not be true of many market offerings. Therefore, the expansion of the current framework to model multiple-usage contexts for product family design is one of the interesting 
directions for future work. Also, the proposed framework creates performance models and preference models in two separate steps (Phase III and Phase IV, respectively), in which error from lower level model may cause issues in upper level model estimation. Hence, the all-in-one Hierarchical Bayesian choice modelling approach [18] can be used to improve the stability of model estimation in the proposed framework. Furthermore, the potential influence of usage context upon the choice set construction in a two-stage decision making process [53] would be another interesting research topic. Lastly, the introduction of the social impact into the hybrid electric vehicle example would bring new light to understanding customers' choice behaviour and attitude towards new technology.

\section{ACKNOWLEDGEMENT}

Grant support from National Science Foundation (CMMI-0700585 and DUE-0920047) is greatly appreciated.

\section{REFERENCES}

[1] Li, H., and Azarm, S., 2000, "Product Design Selection under Uncertainty and with Competitive Advantage," Transactions of ASME: Journal of Mechanical Design, 122(4), pp. 411-418.

[2] Besharati, B., Azarm, S., and Farhang-Mehr, A., "A Customer-Based Expected Utility Metric for Product Design Selection," Proc. Proceedings of the 2002 ASME Design Engineering Technical Conferences and Computers in Engineering Conference.

[3] Wassenaar, H. J., and Chen, W., 2003, "An Approach to Decision-Based Design With Discrete Choice Analysis for Demand Modeling," Journal of Mechanical Design, 125(3), pp. 490-497.

[4] Michalek, J. J., 2005, "Preference Coordination in Engineering Design Decision-Making,"PhD. Dissertation, University of Michigan, Ann Arbor, MI.

[5] Michalek, J. J., Ceryan, O., Papalambros, P. Y., and Koren, Y., 2005, "Manufacturing Investment and Allocation in Product Line Design Decision Making," Proceedings of the 2005 ASME Design Engineering Technical Conferences and Computers in Engineering ConferenceLong Beach, CA, pp. DETC2005-84812.

[6] Kumar, D., Kim, H., and Chen, W., 2006, "Multi-level Optimization for Enterprise Driven Decisionbased Product Design," Decision Making in Engineering Design, K. Lewis, W. Chen, and L. Schmidt, eds., ASME Press, New York.

[7] Sullivan, E., Ferguson, S., and Donndelinger, J. A., 2011, "Exploring Differences in Preference Heterogeneity Representation and Their Influence in Product Family Design," Proceedings of the 2011 ASME Design Engineering Technical Conferences and Computers in Engineering ConferenceWashington, DC, pp. DETC2011-48596.

[8] Turner, C., Ferguson, S., and Donndelinger, J. A., 2011, "Exploring Heterogeneity of Customer Preference to Balance Commonality and Market Coverage," Proceedings of the 2011 ASME Design Engineering Technical Conferences and Computers in Engineering ConferenceWashington, DC, pp. DETC2011-48581.

[9] Frischknecht, B. D., Whitefoot, K., and Papalambros, P. Y., 2010, "On the Suitability of Econometric Demand Models in Design for Market Systems," Journal of Mechanical Design, 132, p. 121007.

[10] Shiau, C. S. N., and Michalek, J. J., 2009, "Should designers worry about market systems?," Journal of Mechanical Design, 131, p. 011011. 
He L., Chen W., Hoyle C., Yannou B., (2012), Choice modeling for usage context-based design. Journal of Mechanical Design, vol. 134(3), doi. DOI: 10.1115/1.4005860.

[11] Michalek, J. J., 2004, "A study of fuel efficiency and emission policy impact on optimal vehicle design decisions," Journal of Mechanical Design, 126, p. 1062.

[12] Michalek, J. J., Ceryan, O., and Papalambros, P. Y., 2006, "Balancing marketing and manufacturing objectives in product line design," Journal of Mechanical Design, 128, p. 1196.

[13] Williams, N., Azarm, S., and Kannan, P., 2008, "Engineering product design optimization for retail channel acceptance," Journal of Mechanical Design, 130, p. 061402.

[14] Ben-Akiva, M., and Lerman, S. R., 1985, Discrete Choice Analysis: Theory and Application to Travel Demand, MIT Press, Cambridge, MA.

[15] Hausman, J., and McFadden, D., 1984, "Specification tests for the multinomial logit model," Econometrica: Journal of the Econometric Society, pp. 1219-1240.

[16] Koppelman, F. S., and Sethi, V., 2000, "Closed Form Discrete Choice Models," Handbook of Transport Modelling, 1, pp. 211-222.

[17] Train, K. E., 2003, Discrete Choice Methods with Simulation, Cambridge University Press, Cambridge.

[18] Hoyle, C., Chen, W., Wang, N., and Gomez Levi, G., 2010, "Understanding and Modeling Heterogeneity of Human Preferences for Engineering Design," Journal of Engineering Design.

[19] Belk, R., 1974, "An exploratory assessment of situational effects in buyer behavior," Journal of Marketing Research, pp. 156-163.

[20] Dickson, P., 1982, "Person-situation: Segmentation's missing link," The Journal of Marketing, 46(4), pp. 56-64.

[21] De la Fuente, J., and Guillén, M., 2005, "Identifying the influence of product design and usage situation on consumer choice," International Journal of Market Research, 47(6), p. 667.

[22] Robertson, T., and Ward, S., 1973, "Consumer Behavior Research: Promise and Prospects," Consumer behavior: theoretical sources, pp. 3-42.

[23] Lavidge, R., 1966, "The cotton candy concept: Intra-individual variability," Lee Adler \& Irving Crespi. Attitude Research at Sea, pp. 39-50.

[24] Engel, J., Kollat, D., and Blackwell, R., 1969, "Personality measures and market segmentation: Evidence favors interaction view," Business Horizons, 12(3), pp. 61-70.

[25] Belk, R., 1975, "Situational variables and consumer behavior," Journal of Consumer Research, pp. 157-164.

[26] Christensen, C., Cook, S., and Hall, T., 2005, "Marketing malpractice: the cause and the cure," Harvard business review, 83(12), p. 74.

[27] Stefflre, V., 1971, "New Products and New Enterprises: A Report of an Experiment in Applied Social Science," Irvine, CA: University of California.

[28] Ratneshwar, S., and Shocker, A., 1991, "Substitution in use and the role of usage context in product category structures," Journal of Marketing Research, 28(3), pp. 281-295.

[29] Berkowitz, E., Ginter, J., and Talarzyk, W., "An investigation of the Effects of Specific Usage Situations on the Prediction of Consumer Choice Behavior," Proc. Educators' Proceedings, B. Greenberg, and D. Bellenger, eds., American Marketing Association. 
He L., Chen W., Hoyle C., Yannou B., (2012), Choice modeling for usage context-based design. Journal of Mechanical Design, vol. 134(3), doi. DOI: 10.1115/1.4005860.

[30] Ulrich, K., and Eppinger, S., 2003, Product design and development, Irwin Professional Pub.

[31] Green, M. G., Palani, R. P. K., and Wood, K. L., "Product Usage Context: Improving Customer Needs Gathering and Design Target Setting," Proc. 2004 ASME Design Engineering Technical Conference, pp. DETC/DTM2004-57498.

[32] Green, M. G., Tan, J., Linsey, J. S., Seepersad, C. C., and Wood, K. L., "Effects of Product Usage Context on Consumer Product Preferences," Proc. 2005 IDETC/CIE Conference, pp. DETC200585438 .

[33] Green, M. G., Linsey, J. S., Seepersad, C. C., Wood, K. L., and Jensen, D. J., "Frontier Design: A Product Usage Context Method," Proc. 2006 ASME Design Engineering Technical Conference, pp. DETC/DTM 2006-99608.

[34] He, L., Chen, W., and Conzelmann, G., 2011, "On Usage Context of Hybrid Electric Vehicle in Choice Studies," Proceedings of the 2011 ASME Design Engineering Technical Conferences and Computers in Engineering ConferenceWashington, DC, pp. DETC2011-48385.

[35] Goldberg, P. K., 1995, "Product differentiation and oligopoly in international markets: The case of the US automobile industry," Econometrica: Journal of the Econometric Society, pp. 891-951.

[36] Simonson, I., and Tversky, A., 1992, "Choice in context: Tradeoff contrast and extremeness aversion," Journal of Marketing Research, 29(3), pp. 281-295.

[37] Kroes, E. P., and Sheldon, R. J., 1988, "Stated preference methods: an introduction," Journal of Transport Economics and Policy, 22(1), pp. 11-25.

[38] Samuelson, P. A., 1948, "Consumption theory in terms of revealed preference," Economica, 15(60), pp. 243-253.

[39] Louviere, J. J., Hensher, D. A., and Swait, J. D., 2000, Stated Choice Methods: Analysis and Application, Cambridge University Press, New York.

[40] Hoyle, C., Chen, W., Ankenman, B., and Wang, N., 2009, "Optimal Experimental Design of Human Appraisals for Modeling Consumer Preferences in Engineering Design," Journal of Mechanical Design, 131(7), pp. 98-107.

[41] Yannou, B., Wang, J., and Yvars, P., 2010, "Computation of the usage contexts coverage of a jigsaw with CSP techniques," 2010 ASME Design Engineering Technical ConferencesMontreal, Canada.

[42] McCullagh, P., 1980, "Regression Models for Ordinal Data," Journal of the Royal Statistical Society. Series B (Methodological), 42(2), pp. 109-142.

[43] Koppelman, F., and Bhat, C., 2006, "A Self Instructing Course in Mode Choice Modeling: Multinomial and Nested Logit Models," U.S. Department of Transportation.

[44] Hartigan, J. A., and Wong, M. A., 1979, "Algorithm AS 136: A k-means clustering algorithm," Journal of the Royal Statistical Society. Series C (Applied Statistics), 28(1), pp. 100-108.

[45] He, L., and Chen, W., 2011, "Usage Context-based Choice Modeling for Hybrid Electric Vehicles," International Conference on Engineering DesignCopenhagen, Denmark, p. Contribution 155.

[46] Yannou, B., Wang, J., Rianantsoa, N., Hoyle, C., Drayer, M., Chen, W., Alizon, F., and Mathieu, J.P., 2009, "Usage Coverage Model For Choice Modeling: Principles And Taxonomy," 2009 ASME Design Engineering Technical ConferencesSan Diego, CA.

[47] Corporation, S., 1996-2009, "StataSE 9.2,"College Station, Texas. 
[48] Ehsani, M., Gao, Y., and Emadi, A., 2009, Modern electric, hybrid electric, and fuel cell vehicles: fundamentals, theory, and design, CRC.

[49] Axsen, J., Burke, A., and Kurani, K., 2008, "Batteries for Plug-in hybrid electric Vehicles (PHEVs): Goals and the state of Technology circa 2008," Institute of Transportation Studies, University of California Davis, Davis, CA.

[50] Shiau, C., Samaras, C., Hauffe, R., and Michalek, J., 2009, "Impact of battery weight and charging patterns on the economic and environmental benefits of plug-in hybrid vehicles," Energy Policy, 37(7), pp. 2653-2663.

[51] EPA, U. E. P. A., 2008, "Fuel Economy Guide 2008."

[52] He, L., Hoyle, C., and Chen, W., 2010, "Examination of customer satisfaction surveys in choice modelling to support engineering design," Journal of Engineering Design, 99999(1), pp. 1-19.

[53] Hauser, J. R., Toubia, O., Evgeniou, T., Befurt, R., and Dzyabura, D., 2010, "Disjunctions of Conjunctions, Cognitive Simplicity and Consideration Sets," Journal of Marketing Research, 47(3), pp. 485-496. 